\title{
A Comparison of the Histopathology of Premalignant and Malignant Mammary Gland Lesions Induced in Sexually Immature Rats with those Occurring in the Human
}

\author{
Meenakshi Singh, John N. McGinley, and Henry J. Thompson \\ Department of Pathology (MS), University of Colorado Health Sciences Center; and Center for Nutrition in the \\ Prevention of Disease (JNM, HJT), AMC Cancer Research Center, Denver, Colorado
}

\begin{abstract}
SUMMARY: The injection of sexually immature female rats with 1-methyl-1-nitrosourea results in a rapid induction of premalignant and malignant mammary gland lesions within 35 days of carcinogen administration. This model affords the opportunity for investigators to study the process of mammary carcinogenesis over a very short latency and to investigate early events in this process. We have recently published on various aspects of this system including the histology of the lesions induced, the time frame of their occurrence, and their dependence on ovarian hormones for their maintenance and growth. In this report we present evidence that many aspects of the histopathology of mammary lesions in this model system are similar to those occurring in humans. We also discuss aspects of the human disease, which are not recapitulated in this model. (Lab Invest 2000, 80:221-231).
\end{abstract}

$A$ $\mathrm{n}$ animal model system for studying premalignant and malignant stages of breast cancer induced in immature female rats has been reported (Thompson et al, 1995). This model has characteristics that distinguish it from other chemically induced systems in the rat (Clark, 1996; Russo et al, 1990a; Welsch, 1985). Among these is the rapidity with which lesions occur in essentially all animals in an experimental group and the ability to detect large numbers of premalignant lesions (Thompson et al, 1995). We have also recently reported on the temporal sequence of lesion occurrence and the frequency of lesion occurrence in the absence of the ovaries (Thompson et al, 1998a; 1998b; Kelloff et al, 1998). One aspect of the model that has not been reported in detail is how the histology of the lesions that occur compares to lesions observed in the human breast. In this article we apply the same criteria used to evaluate lesions observed in the human breast (Page and Anderson, 1987; Page and Rogers, 1992; Rogers, 1987; Rubin and Simpson, 1998; Tavassoli, 1992; Tavassoli and Norris 1990) to the diagnosis of lesions in this model system. The similarities and the differences in the morphology of mammary lesions induced in the rat in our short-

Received November 2, 1999.

Supported by Public Health Service grant NO1-CN-55178 from the National Cancer Institute.

Address reprint requests to: $D r$. H. J. Thompson, AMC Cancer Research Center, 1600 Pierce Street, Lakewood, Colorado 80214. Fax: 303239 3443; E-mail: thompsonh@amc.org duration chemical carcinogenesis model and human breast pathology are reported.

\section{Results}

\section{Normal and Hyperplasia}

Mammary lobular acini in rats, as in humans, are lined by a single layer of luminal epithelial cells and a somewhat discontinuous layer of flattened myoepithelial cells (Fig. 1, R1). In younger animals terminal end buds (TEB) are seen. TEB are comprised of dark cells, intermediate cells, and myoepithelial cells (Fig. 1, R2). Mitoses are frequent. TEB have a single lumen with a smooth border.

In the short-duration carcinogenesis model developed by us, a spectrum of proliferative lesions has been observed (Thompson et al, 1995). In this model hyperplasia ranged the full gamut from mild to florid. In a single field, varying grades of hyperplasia were observed. The primary criterion used for diagnosing hyperplasia was an increase in the layers of epithelial cells lining the acini and ducts. In mild hyperplasia, this varied from three to four cells thick (Fig. 1, R3). Criteria for moderate hyperplasia were more than four cells thick with an occasional broad bridge of epithelium across the lumen (Fig. 1, R4). In florid hyperplasia, ducts were packed with cells, and the primary lumen, though still identifiable, was slit like and displaced toward the periphery with some expansion of the circumference of the ductal structure and formation of irregular secondary lumina (Fig. 1, R5). In florid hyperplasia, cells showed streaming and swirling and there was nuclear overlap. The nuclei of the cells in the 


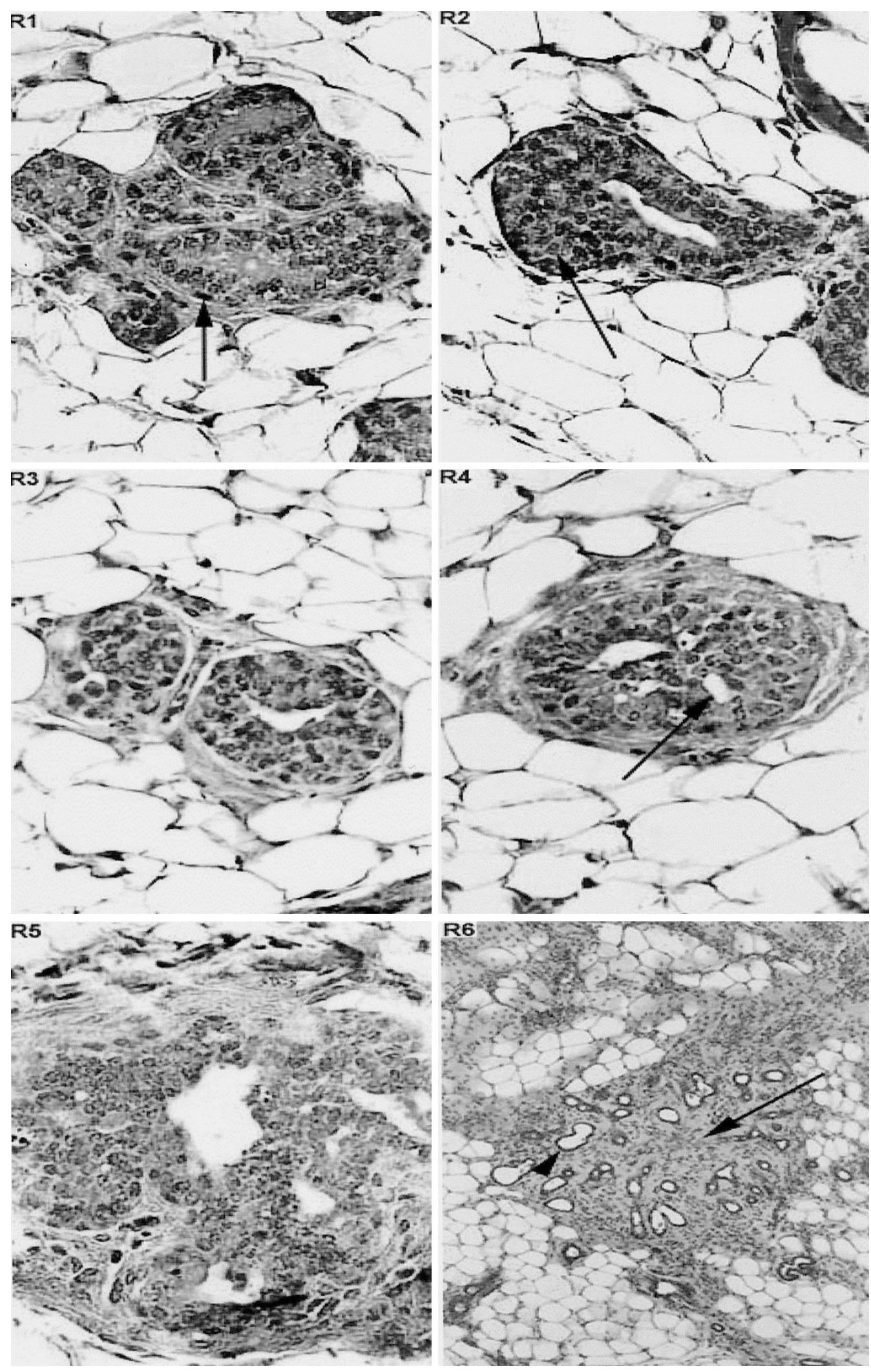

\section{Figure 1.}

R1, Normal lobuloalveolar unit. The acini are lined by a layer of cuboidal luminal cells and a discontinuous layer of flat myoepithelial cells (arrow). R2, Normal termina end bud (TEB). Heterogenous population of dark cells, intermediate cells (arrow), and myoepithelial cells. The luminal border is smooth and mitoses are frequent. R3, Mild hyperplasia. Three- to four-cell thick epithelium and comprised of intermediate cells. R4, Moderate hyperplasia. More than four-cell thick epithelium and secondary lumina (arrow). R5, Florid epithelial hyperplasia. The duct is expanded and filled with haphazardly arranged cells. Secondary lumina are present. R6, Fibroadenoma (low power). Tumor with proliferation of epithelial (arrowhead) and stromal (arrow) elements. Irregular edges. R7, Fibroadenoma (high power of R6). 

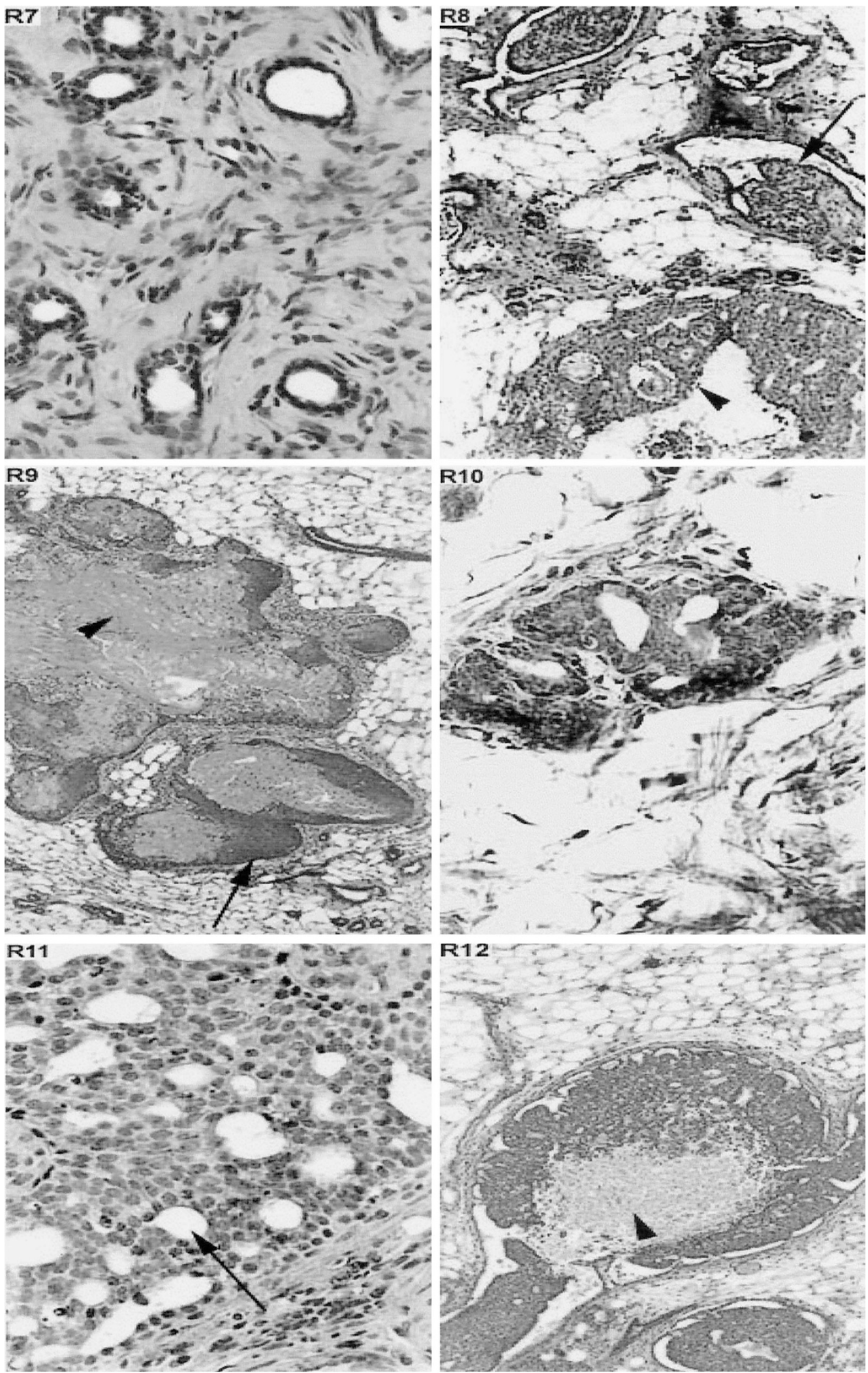

Figure 1. Continued.

The nuclei are benign. R8, Micropapillomas (arrow) adjacent to carcinoma in situ (CIS) (arrowhead). R9, Epidermal inclusion cyst. Cyst lined by stratified squamous epithelium (arrow) and filled with keratin debris (arrowhead). R10, Lobuloalveolar unit with earliest change in the form of plump epithelial lining without significant nuclear changes. R11, cribriform CIS. Duct filled with a uniform population of cells with regularly arranged, round to oval, hyperchromatic nuclei. The secondary lumina have a clean punched out appearance (arrow). R12 and R13, Ductal carcinoma in situ (DCIS). Low and high power appearance of an expanded duct filled with neoplastic cells that resemble those in cribriform DCIS. Although central necrotic material is present (arrowhead), high-grade nuclear features are absent. R14, CIS with mixed papillary (arrowhead) and cribriform (arrow) features. R15 (Low power) and R16 (high power), Cribriform adenocarcinoma with a few mature blood vessels (arrow) 

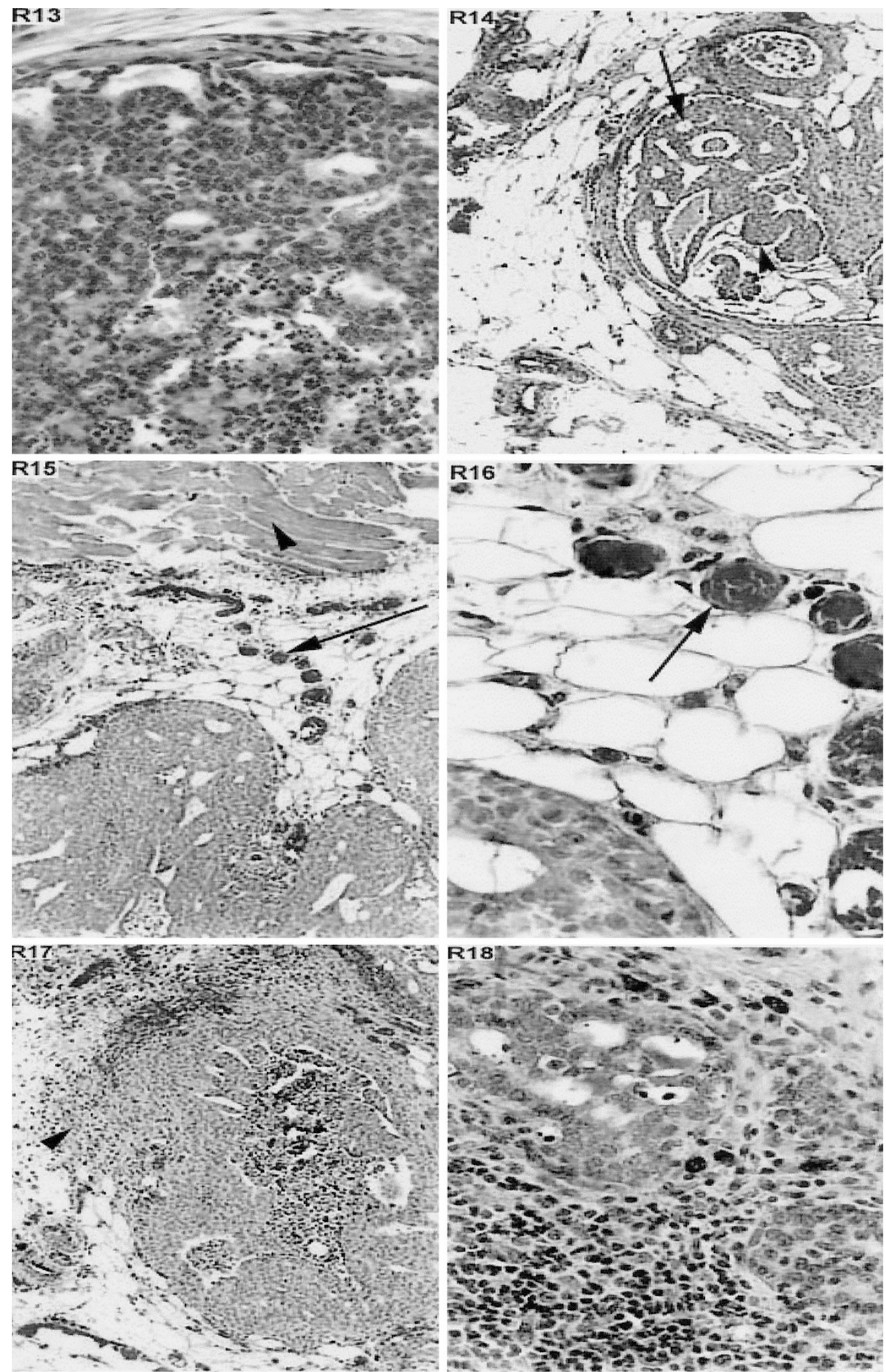

Figure 1. Continued.

at the periphery where it is invading muscle (arrowhead) in R15. R17, Ductal carcinoma. Earliest evidence of invasion with breach of basement membrane of the duct (arrowhead). R18 (High power), Adenocarcinoma (arrow) involving a lymph node. R19, Rat mammary gland whole mount. Multiple lesions that had varied histology. R20, Subgross of the lesion, an adenocarcinoma, in the square in R19. 


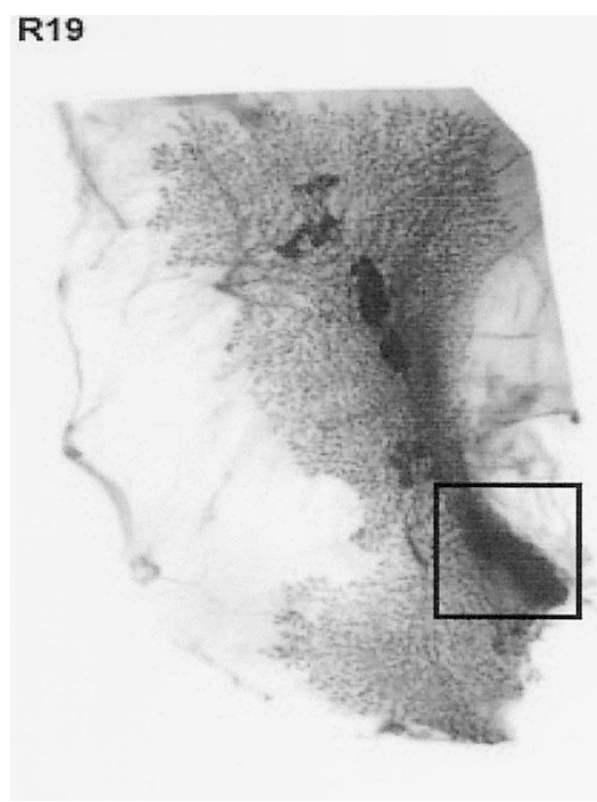

Figure. 1. Continued.

hyperplastic lesions were characteristically round to oval and generally lacked nucleoli. Often there was a haphazard arrangement of the nuclei. Individual cell outlines were not discernible (Fig. 1, R5). Hyperplastic foci were also frequently present in the vicinity of carcinomatous foci. In humans, hyperplasia of the usual type has a similar histology (Fig. 2, H1). Other types of hyperplasia that are seen in human breast specimens, eg, hypersecretory hyperplasia and gynecomastoid hyperplasia, have not been seen by us in rat mammary glands. This list of dissimilarities can be further expanded to include other benign entities such as sclerosing adenosis, complex sclerosing lesion, apocrine change, and collagenous spherulosis.

\section{Benign Tumors}

Fibroadenomas, micropapillomas, ie, microscopic papillomas, and epidermal inclusion cysts were the benign breast tumors seen by us in this rat model. Fibroadenomas displayed proliferation of epithelium and stroma (Fig. 1, R6). The edges were irregular, lesions were not well circumscribed, and the stromal component was less well developed than the fibroadenomas seen in humans or in the longer term rat carcinogenesis models. The nuclei lacked features of anaplasia and were rather innocuous (Fig. 1, R7). Unlike humans, in which fibroadenomas may on occasion display foci of atypical ductal hyperplasia (ADH), atypical lobular hyperplasia, or carcinoma in situ (CIS), the rat sections did not display these within the confines of fibroadenomas. As in humans (Fig. 2, $\mathrm{H} 2$ and $\mathrm{H} 3$ ), some degree of epithelial hyperplasia was observed by us in rat fibroadenomas. We have not observed phyllodes tumor in the rat. Benign micropapillomas have been seen by us in this rat model. These were composed of papillary proliferations with fibrovascular cores, within cystic spaces (Fig. 1, R8,

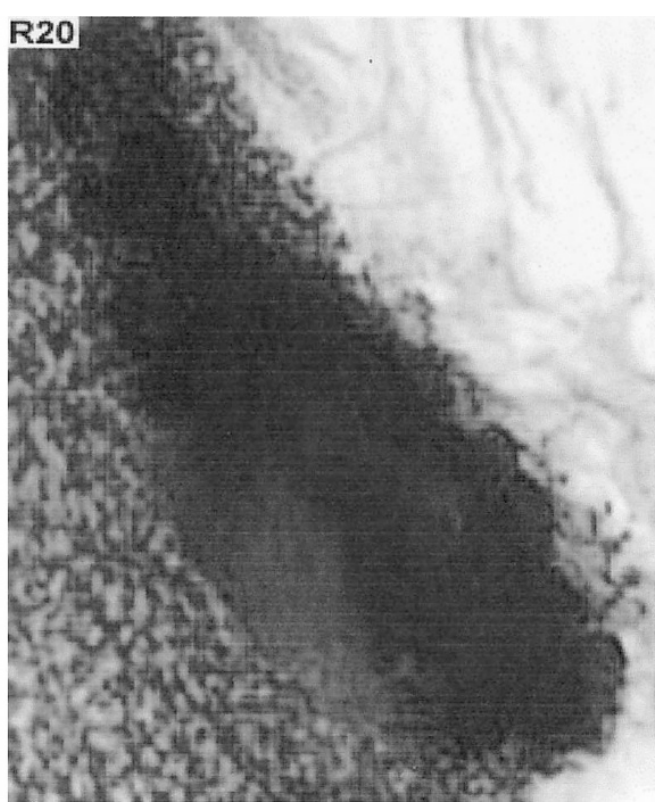

arrow). The papillae were lined by benign appearing epithelium that focally was hyperplastic. Epidermal inclusion cysts (Fig. 1, R9) were lined by benign stratified squamous epithelium and filled with keratin debris. In rats these were seen to arise both in the overlying skin and within mammary lobules.

\section{CIS}

In our short-duration model, we have observed a high frequency of CIS. Combined cytologic and architectural criteria were used in diagnosing CIS. Keeping in mind that these lesions were being dissected at a very early stage after carcinogen (35 days), for the diagnosis of CIS, we required that at least one expanded ductal structure be completely replaced by neoplastic cells that had uniform, monotonous, round, hyperchromatic, and non-overlapping nuclei. We are aware of the present dilemma that surgical pathologists oftentimes face when trying to distinguish $\mathrm{ADH}$ from minimal involvement by $\mathrm{CIS}$. In the rat carcinogenesis model, however, it has been our experience that if the postcarcinogen phase of the experiment is prolonged, as in conventional rat carcinogenesis models (Thompson and Adlakha, 1991), essentially all the animals develop multiple adenocarcinomas. Therefore, we thought it prudent that these single-space lesions be classified as $\mathrm{CIS}$ without a requirement for the involvement of two-duct cross-sections by neoplastic cells (Page and Rogers, 1992) or for the lesion to be $2 \mathrm{~mm}$ or more (Tavassoli, 1992) before labeling them as CIS-as is conventionally applied to differentiate ADH from ductal carcinoma in situ (DCIS) in humans. Unlike in humans (Fig. 2, H4) we did not observe microcalcifications in the rat lesions. No atypical lobular hyperplasia was observed by us in the rats. We did notice that some of the lobular units showed acinar structures with plump epithelial lining without any signifi- 

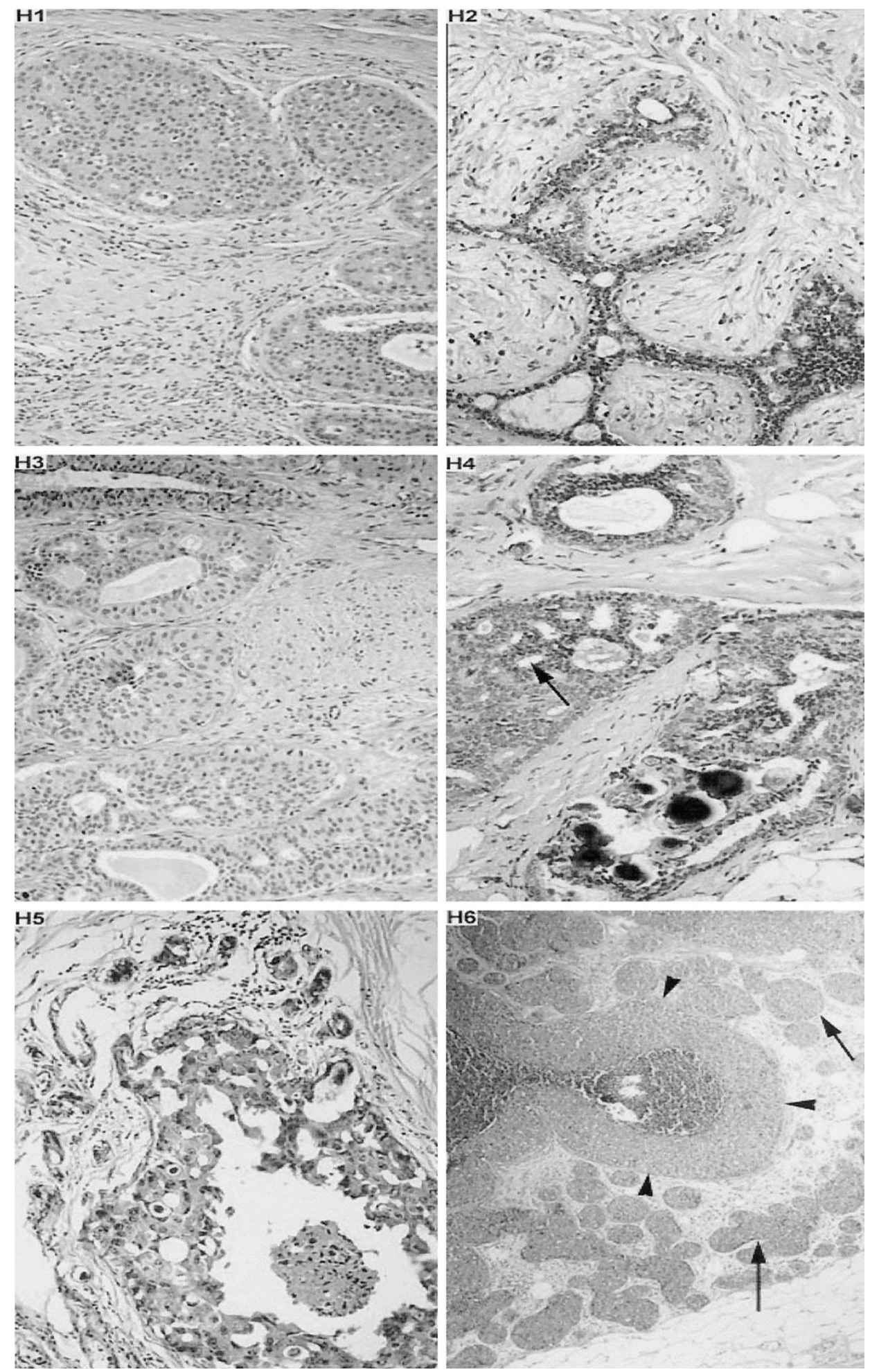

\section{Figure 2.}

H1, Florid compact hyperplasia of the usual type. Ducts compactly filled with epithelial cells with oval nuclei that are haphazardly spaced (low power). H2, Fibroadenoma. Benign neoplasm with epithelial and stromal proliferation. The epithelium has been compressed into strands. Nuclei lack anaplastic features and are bland. H3, Florid epithelial hyperplasia within a fibroadenoma. H4, Cribriform DCIS. Expanded ducts filled with uniform cells with round, hyperchromatic nuclei and punched out secondary spaces (arrow). Microcalcifications are evident. H5, Comedo DCIS. CIS with central necrosis and multi-layered ductal epithelial lining with highly pleomorphic cells demonstrating high-grade nuclei. H6 (Low power), Comedo DCIS with cancerization of lobules. The neoplastic cells are extending from the ducts (arrowheads) into lobules (arrows). H7, Invasion. Single carcinoma cells in a so-called Indian file pattern of invasion into the stroma (arrows). H8, Lobular CIS. Entire lobular unit is distended, distorted, and packed with a uniform population of cells. H9, Lymph node (upper one third) with metastasis of lobular carcinoma (lower two thirds). There is extension into extranodal tissue. 

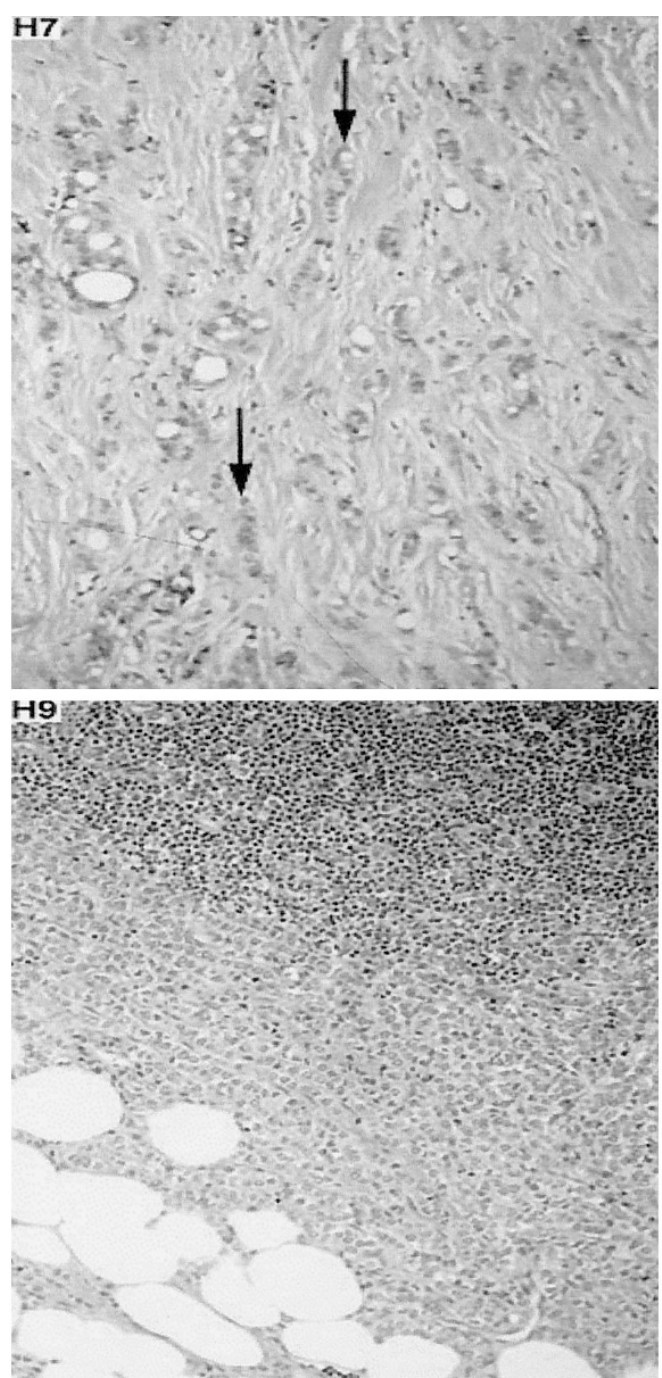

Figure 2. Continued.

cant increase in the number of cells lining them or a change in the nuclear characteristics (Fig. 1, R10). Of course in cycling animals the acinar epithelium does show intracytoplasmic vacuoles and luminal secretions in response to the hormonal changes of the estrous cycle. There was no intracytoplasmic vacuolization in the hyperplastic epithelium.

The most frequent subtype of CIS in our series of rat experiments was the cribriform type (Fig. 1, R11). The criteria used by us for diagnosing cribriform CIS was that at least one expanded ductal lumen had to be completely replaced by a monotonous population of cells with round, hyperchromatic, and nonoverlapping nuclei. No swirling was to be observed. Architectural distortions with clean punched out spaces, rigid bridges, and the so-called Roman arch formation could also be present. There should be no remnants of the primary lumen.

For the diagnosis of comedo CIS, the cells had to have high-grade anaplastic nuclei, ie hyperchromasia, pleomorphism, irregular nuclear membranes and nucleoli, with central necrosis. Although some of the cribriform DCIS foci had central necrosis, the high-

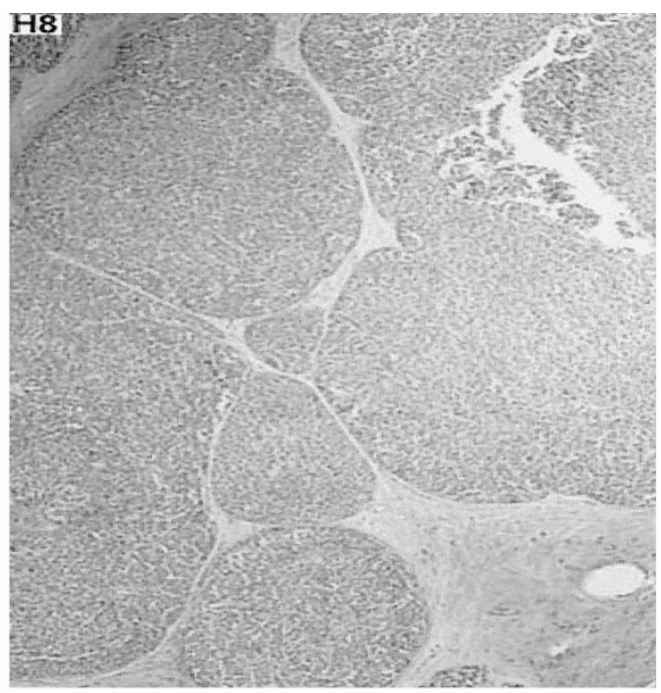

grade nuclei needed for diagnosing comedocarcinoma in humans (Fig. 2, H5 and H6) have not been noted by us in the rat lesions (Fig. 1, R12 and R13). Therefore, classic comedocarcinoma in situ was not a feature of lesions induced in this rat model. Cancerization of lobules, ie, extension of the carcinoma cells within lobules, a frequent finding in cases of comedo $\mathrm{CIS}$ in humans (Fig. 2, H6), has also not been seen by us in the rat model.

Occasionally a papillary CIS was observed. When papillary processes were observed within a CIS, it was categorized as a papillary CIS. These had nuclei similar to those of cribriform CIS. The morphology of these lesions in the rat was similar to the lesions in humans. A mixture of patterns was identified in some of the CIS in rats (Fig. 1, R14), and this may also be seen in humans. The pure micropapillary pattern has not been observed by us in the rat and neither has apocrine DCIS, subtypes that are reported in humans. In all cases of CIS, the basement membrane of the ducts was intact. A desmoplastic stroma with a lymphocytic infiltrate admixed with mast cells was observed around some of the CIS ducts. Histologic 
features that are demonstrated by breast carcinoma in humans but have not been seen in our model include elastosis around neoplastic ducts and microcalcifications.

\section{Adenocarcinoma}

In the rat the size of the adenocarcinoma was quite variable. At necropsy, detection of smaller lesions was facilitated by the location of congested small vessels with the help of low-magnification eye pieces. Regions adjacent to such foci were considered suspicious and were dissected for histologic scrutiny. On light microscopy we frequently observed small, but mature, blood vessels with thin walls in and around the periphery of the foci of CIS and adenocarcinoma (Fig. 1, R15 and R16). However, this is not exclusive to malignant lesions because similar vessels were seen by us in histologically normal mammary parenchyma. The density of these vessels was not markedly increased.

The earliest evidence of invasion was observed as a breach of the basement membrane of the ducts (Fig. 1, R17). Rat mammary adenocarcinomas do not tend to invade as single cells but more on a broad front and with clusters of neoplastic acini. In humans a singlecell pattern of invasion is often noted (Fig. 2, H7).

The subtypes of invasive adenocarcinoma seen by us in the rat were the cribriform, papillary, and no special type. Special types of carcinoma that are seen in humans and that were not observed in the rat model include: pure tubular carcinoma, colloid/mucinous carcinoma, adenoid cystic carcinoma, and medullary carcinoma or its variant. Although a lymphocytic response was observed in a few of the carcinomas, they did not have the anaplastic nuclear features that characterize a medullary carcinoma. Lobular carcinoma is common in humans (Fig. 2, H8), but no lobular $\mathrm{CIS}$ or invasive lobular carcinoma was observed in the rat.

\section{Lymph Node Involvement}

The incidence of lymph node involvement in the rat model is fairly low, though this is occasionally seen. The lymph node metastases were easy to identify in the rat microscopic sections (Fig. 1, R18); this is in sharp contrast to some cases of lymph node metastasis of lobular carcinoma in humans, which may at times involve a lymph node in a subtle manner (Fig. 2, $\mathrm{H} 9)$.

\section{Invasion}

No definite capillary-lymphatic space invasion was observed. Muscle invasion was noted in the rat (Fig. 1, R15). We have not observed perineural invasion in the rats.

\section{Other Tumors}

Metaplastic carcinomas have not been seen by us in the rat and neither have other stromal lesions like fibromatosis and fibrosarcoma.

\section{Discussion}

A rat model for mammary carcinogenesis of short latency has been reported (Thompson et al, 1995). This model seems to have numerous advantages, including its use in the rapid evaluation of compounds for chemopreventive activity as well as for mechanistic inquiries in which progression of premalignant lesions to carcinomas is of interest (Thompson et al, 1998a, 1998b). Because of the importance of understanding how the pathogenesis of the disease process in the rat relates to that which occurs in the human, we have described the lesions induced in this rat model using the terminology and criteria applied to human breast diseases (Page and Anderson, 1987; Page and Rogers, 1992; Rogers, 1987; Rubin and Simpson, 1998; Tavassoli, 1992; Tavassoli and Norris, 1990). This approach differs from that used to classify mammary gland lesions induced in the rat by other carcinogens or by using the same carcinogen administered with different carcinogen-dosing regimens (Russo et al, $1990 b)$. In this article we applied those criteria used in diagnosing human breast pathology to the lesions observed in the rat mammary gland in response to the injection of the carcinogen 1-methyl-1-nitrosourea (MNU) at 21 days of age. The similarities and dissimilarities observed between the rat lesions and human breast pathology are summarized in Table 1.

\section{Hyperplasia}

The hyperplastic lesions observed in the rat are in our opinion best classified as hyperplasia of the usual type, and in general are comparable to the types of hyperplasia observed in the human breast. Unlike some reports in the literature, we did observe secondary lumina in moderate or florid hyperplasias (Russo et al, 1990b). Interestingly we have not seen atypical lobular hyperplasia in our rat experiments. The reasons for this are unclear. The frequent observation of coexisting hyperplasia and adenocarcinoma in the rats as in humans supports recently published evidence that these lesions are the precursors of carcinoma (Thomspon et al, 1998b).

\section{Microcalcifications}

In humans, microcalcifications, although not pathognomonic or exclusive, are a common feature of DCIS or invasive carcinoma. This has proved to be a useful radiologic feature in mammographic surveillance and detection of breast cancer, postmammography triaging of women for breast aspiration or biopsy, and follow-up for recurrence of cancer (Rubin and Simpson, 1998). It is noteworthy, although not particularly surprising, that no microcalcifications were detected in the rat model. The explanation for this may lie in the early detection and specimen procurement in our rat model. As calcifications are often a tombstone of sort, the lesions in our model may not be "old enough" to have developed them. A similar explanation can be 
Table 1. Summary of Characteristics of Breast Lesions Observed in the Human Versus the Rat

\begin{tabular}{|c|c|c|}
\hline & Human & Rat \\
\hline Normal & $\begin{array}{l}\text { - Luminal epithelium and } \\
\text { myoepithelial cell layer }\end{array}$ & Same as human \\
\hline \multirow[t]{3}{*}{ Benign lesions } & $\begin{array}{l}\text { - Fewer epidermal inclusion } \\
\text { cysts }\end{array}$ & $\begin{array}{l}\text { - Epidermal inclusion cysts are } \\
\text { relatively frequent, and occur } \\
\text { within the mammary lobules } \\
\text { also }\end{array}$ \\
\hline & $\begin{array}{l}\text { Fibroadenomas may } \\
\text { occasionally display ADH } \\
\text { and CIS }\end{array}$ & $\begin{array}{l}\text { - No ADH or CIS within } \\
\text { fibroadenomas }\end{array}$ \\
\hline & $\begin{array}{l}\text { - A wide spectrum of benign } \\
\text { lesions occurs }\end{array}$ & $\begin{array}{l}\text { A very limited number of } \\
\text { benign lesions, including } \\
\text { fibroadenoma, papilloma }\end{array}$ \\
\hline Hyperplasia & $\begin{array}{l}\text { - Special types of hyperplasia } \\
\text { may occur }\end{array}$ & $\begin{array}{l}\text { - Hyperplasia of the usual type is } \\
\text { seen; no special types of } \\
\text { hyperplasia }\end{array}$ \\
\hline ALH & - May be observed & - Not observed \\
\hline \multirow[t]{3}{*}{ DCIS } & $\begin{array}{l}\text { - Many histologic subtypes } \\
\text { of CIS }\end{array}$ & - Cribriform and papillary DCIS \\
\hline & & $\begin{array}{l}\text { - No true micropapillary, comedo } \\
\text { and apocrine DCIS }\end{array}$ \\
\hline & $\begin{array}{l}\text { - There may be elastosis and } \\
\text { microcalcifications }\end{array}$ & $\begin{array}{l}\text { - No elastosis and } \\
\text { microcalcifications }\end{array}$ \\
\hline \multirow[t]{3}{*}{ Carcinoma } & $\begin{array}{l}\text { - There may be a single file } \\
\text { pattern of invasion by } \\
\text { carcinoma cells }\end{array}$ & $\begin{array}{l}\text { Broad front of stromal } \\
\text { invasion, and not as single } \\
\text { cells }\end{array}$ \\
\hline & $\begin{array}{l}\text { - Many special types of } \\
\text { carcinomas }\end{array}$ & $\begin{array}{l}\text { - No tubular, mucinous, adenoid } \\
\text { cystic, medullary and lobular } \\
\text { carcinoma }\end{array}$ \\
\hline & $\begin{array}{l}\text { - Lymph node involvement is } \\
\text { frequent }\end{array}$ & $\begin{array}{l}\text { - Lymph node involvement is } \\
\text { infrequent }\end{array}$ \\
\hline
\end{tabular}

put forward for there being only mild desmoplasia around some DCIS lesions.

\section{CIS}

We found CIS in the rat model, a finding consistent with other reports (Crist et al, 1992; Russo et al, 1990b). Although the histologic spectrum of CIS observed in the rat was limited compared with that seen in humans, the lesion types observed in rats represent commonly observed types in humans. Although pure micropapillary DCIS was not seen, there was some attempt at luminal micropapillae formation in ducts that showed cribriform DCIS.

\section{Carcinomas}

We did not observe in the rat many of the histologic subtypes of carcinoma that are seen in humans. In humans these subtypes are associated with differing prognosis, eg, tubular carcinoma is associated with a good prognosis. One finding that warrants repetition is the absence of histologic features of lobular carcinoma in this rat model. In our experience, this is true both for the short-duration as well as the conventional rat carcinogenesis model (Clark, 1996; Russo et al, 1990a; Thompson et al, 1995; Welsch, 1985).

\section{Invasion and Metastasis}

Other than an occasional focus of adenocarcinoma in the lymph nodes, distant metastases were not observed in the short-duration carcinogenesis model. Nonetheless, we considered the observance of such lesions remarkable given that invasion occurred within 35 days of carcinogen administration and only 3 weeks after the emergence of hyperplasia. It is well known that conventional chemically induced carcinogenesis protocols in the rat do not generate a significant proportion of distant metastases. Given these observations we have pursued the induction of metastases. In a separate experiment, a higher percentage of distant metastases have been induced when multiple doses of carcinogen were injected in young rats and the experiment was prolonged over several months (data not included). Most of these metastases were observed in the lungs. Thus the evidence currently available suggests that lesions induced in the rat using the short time frame approach have aggressive characteristics as do their human counterparts.

\section{Difficulties in Classifying the Rat Lesions}

TEB have to be differentiated from mild hyperplasia. TEB were categorized according to the criteria laid out 
by Russo et al (1990a). They were differentiated from mild hyperplasia by the presence of a heterogenous population of cells, whereas the hyperplastic lesions only had a single cell type, ie, intermediate cells. Like other investigators, we agree that the assessment of invasion in rat mammary carcinomas is difficult at times. In the early lesions that were nonpalpable at the time of necropsy, we assessed invasion by the absence of basement membrane around ducts otherwise showing features of DCIS and the presence of tongues of neoplastic epithelium projecting out from these ducts into the stroma. Once the adenocarcinoma reached palpable size, gross features of a fleshy, necrotic, hemorrhagic mass were helpful in identifying adenocarcinomas. Many of the adenocarcinomas had a predominant DCIS component.

\section{Summary}

The histology of various benign, premalignant, and malignant lesions produced by carcinogen administration to rats for evaluating mammary carcinogenesis is quite similar to that seen in breast biopsy specimens from humans, although a somewhat limited number of benign lesions and subtypes of DCIS and adenocarcinoma are seen in the rat model. We classified the lesions using the same terminology and wellestablished criteria set forth by breast experts for human breast disease and recommend this approach for investigators utilizing the rat mammary carcinogenesis model, so that the interpretation of data generated from these experiments has more relevance in translational research.

\section{Materials and Methods}

\section{Rodent Model}

Female Sprague Dawley rats were obtained from Taconic Farms (Germantown, NY) at 20 days of age. At 21 days of age, animals were injected ip with $50 \mathrm{mg}$ MNU/kg body weight as previously described by our laboratory (Thompson et al, 1995). Rats were housed three per cage in an environmentally controlled room maintained at $22^{\circ} \mathrm{C}$ and $50 \%$ relative humidity with a 12-hour light-dark cycle. They were fed a purified diet and distilled water ad libitum. Animals were euthanized by gaseous $\mathrm{CO}_{2}$ inhalation at 35 days after carcinogen. At necropsy, rats were skinned and the skin was examined under translucent light. The cervical-thoracic and abdominal-inguinal mammary glands were carefully excised and spread onto clean $50 \times 75-\mathrm{mm}$ prelabeled microscope slides. These whole mounts were processed for evaluation and photographed as previously described (Thompson et al, 1995) (Fig.1, R1 and R2). Thereafter all lesions detected by inspection of whole mounts at $\times 2$ magnification were dissected using the photograph to provide a permanent identification record of the location and gross morphology of a lesion. Dissected lesions were processed and histologically classified according to the criteria detailed in the "Results" section.

\section{Human Breast Sections}

The human breast pathology observations were made on microscopic slides from the Breast Consult Service of Dr. David Page at the University of Vanderbilt Medical Center, Nashville, Tennessee and from the Surgical Pathology Section of the Department of Pathology at the University of lowa Hospitals and Clinics.

\section{Acknowledgements}

We thank Dr. David L. Page and Dr. Roy Jensen, Vanderbilt University Medical Center, Nashville, TN, for reviewing the manuscript and for their expert comments.

\section{References}

Clark R (1996). Animal models of breast cancer: Their diversity and role in biomedical research. Breast Cancer Res Treat 39:1-6.

Crist KA, Chaudhuri B, Shivaram S, and Chaudhuri PK (1992). Ductal carcinoma in situ in rat mammary gland. J Surg Res 52:205-208.

Page DL and Anderson TJ (1987). Diagnostic histopathology of the breast. Edinburgh: Churchill Livingstone.

Page DL and Rogers LW (1992). Combined histologic criteria for the diagnosis of mammary atypical ductal hyperplasia. Human Pathol 23:1095-1097.

Rogers LW (1987). Epithelial hyperplasia. In: Page DL and Anderson TJ, editors. Diagnostic histopathology of the breast. New York: Churchill Livingstone, 120-235.

Rubin E and Simpson JF (1998). Microcalcifications in breast specimen radiography. Philadelphia: Lippincott-Raven, 144172.

Russo J, Gusterson BA, Rogers AE, Russo IH, Wellings SR, and vanZwieten MJ (1990a). Biology of disease: Comparative study of human and rat mammary tumorigenesis. Lab Invest 62:244-278.

Russo J, Russo IH, Rogers AE, VanZwieten MJ, and Gusterson B (1990b). Pathology of tumours in laboratory animals. Tumours of the rat. Tumours of the mammary gland. IARC Sci Publ, 99:47-78.

Tavassoli FA (1992). Intraductal hyperplasias, ordinary and atypical in pathology of the breast. New York: Elsevier, 155-191.

Tavassoli FA and Norris HJ (1990). A comparison of the results of long term follow up for atypical intraductal carcinoma of the breast. Cancer 65:518-529.

Thompson HJ and Adlakha $\mathrm{H}$ (1991). Dose responsive induction of mammary gland carcinomas by the intraperitoneal injection of 1-methyl-1-nitrosourea. Cancer Res 51:34113415.

Thompson HJ, McGinley J, Rothhammer K, and Singh M (1995). Rapid induction of mammary intraductal proliferations, ductal carcinoma in situ and carcinomas by the injection of sexually immature female rats with 1-methyl-1nitrosourea. Carcinogenesis 16:2407-2411. 
Thompson HJ, McGinley J, Rothhammer K, and Singh M (1998a). Ovarian hormone dependence of pre-malignant and malignant mammary gland lesions induced in pre-pubertal rats by 1-methyl-1-nitrosourea. Carcinogenesis 19(3):383386.

Thompson HJ, McGinley JN, Wolfe P, Singh M, Steele VE, and Kelloff GJ (1998b). Temporal sequence of mammary intraductal proliferations, ductal carcinomas in situ and adenocarcinomas induced by 1-methyl-1-nitrosourea in rats. Carcinogenesis 19(12):2181-2185.
Welsch CW (1985). Host factors affecting the growth of carcinogen-induced rat mammary carcinomas: A review and tribute to Charles Brenton Huggins. Cancer Res 45:34153443. 